\title{
Magnetic field measurements around massive young stellar objects with the EVN
}

\section{Gabriele SURCIS*}

INAF-Osservatorio Astronomico di Cagliari, Via della Scienza 5, I-09047, Selargius, Italy

E-mail: gabriele.surciseinaf.it

\section{Wouter H.T. VLEMMINGS}

Department of Space, Earth and Environment, Chalmers University of Technology, Onsala Space Observatory, SE-439 92 Onsala, Sweden

E-mail: wouter.vlemmings@chalmers.se

\section{Huib J. van LANGEVELDE}

Joint Institute for VLBI ERIC, Oude Hoogeveensedijk 4, 7991 PD Dwingeloo, The Netherlands Sterrewacht Leiden, Leiden University, Postbus 9513, 2300 RA Leiden, The Netherlands

E-mail: langevelde@jive.eu

\begin{abstract}
Although there has been significant progresses in the last years, the formation process of highmass stars $\left(M>8 M_{\text {sun }}\right)$ is still unclear. This is mainly due to the fast evolution and large distances of the massive young stellar objects (YSOs) that make difficult to observe, with fine details, a sufficient number of massive YSOs at each evolutionary stages. However, in the last 10 years some pieces of information regarding the gas motion and the magnetic field close to the YSOs have been gathered by observing and analyzing the maser emission of mainly water and methanol molecules. In particular, we have performed full polarization observations of $6.7 \mathrm{GHz}$ methanol masers and/or $22 \mathrm{GHz}$ water maser with the European VLBI Network (EVN) towards a large number of sources in order to provide measurements of magnetic fields orientation and strength at milliarcsecond resolution around massive protostars. These measurements can be made at this high angular resolution only by observing and analyzing the polarized emission of masers. From the linearly and circularly polarized emissions we can determine the orientation and the strength of the magnetic field, respectively. Here, we present the updated statistics of the possible alignment between the magnetic field and the bipolar outflows ejected from the massive protostars. In addition, and for the first time, we will provide lower limits of the magnetic field strength measured from the Zeeman-splitting of the methanol maser by using the very recently determined Landé g-factors for the methanol maser transitions. Furthermore, the up-to-date results of the monitoring project of the $22 \mathrm{GHz}$ water masers detected towards the massive star-forming region $\mathrm{W} 75 \mathrm{~N}(\mathrm{~B})$ will be also presented.
\end{abstract}

14th European VLBI Network Symposium \& Users Meeting (EVN 2018)

8-11 October 2018

Granada, Spain

\footnotetext{
* Speaker.
} 


\section{Introduction}

The theoretical and observational advancements of the last decades support the idea that highmass stars $\left(\mathrm{M}>8 \mathrm{M}_{\odot}\right)$ form similarly to low-mass stars. That is the massive young stellar objects (YSOs) are formed through gravitational collapse of cores and during the formation process a disk-outflows system around the central protostar is formed (e.g., [1]; [2]). Several magnetohydrodynamical (MHD) simulations have been carried out recently. For instance, these show that early outflows can reduce the radiation pressure [3], that the collimation of the outflows varies with the time and with the magnetic field strength [4], and that their structure is determined by the large-scale geometry of the magnetic field lines [5]. In particular, the outflows are initially poorly collimated and becomes more collimated only when a nearly Keplerian disk is formed and fast jets are generated [4]. In addition, when the magnetic field is strong the outflow is slow and poorly collimated [4] and when it is weak the outflow is fast and well-collimated [6]. From an observational point of view conflicting results on the orientation of the magnetic field with respect to the outflow orientation have been found. Based on the observations of dust polarized emission (towards 21 sources) no correlation between outflow axis and magnetic field orientation has been found at arcsecond resolution ( 1000 au; [7]), while from the polarized emission of $6.7 \mathrm{GHz}$ methanol masers (19 sources that correspond to the $60 \%$ of our total sample, see next Sect.) we found evidence that on scales of 10-100 au (mas resolution) magnetic field around massive YSOs is preferentially oriented along the outflow [8]. Of great importance for further improving the MHD numerical simulations is to provide new measurements of magnetic fields strength at mas resolution close to the massive YSOs. This has been possible, so far, only through the analysis of the circularly polarized emission of $22 \mathrm{GHz}$ water masers, but because of the shock-nature of these masers, the strength of the magnetic field is estimated in the post-shock compressed gas (e.g., [9]). Although the circularly polarized emission of the $6.7 \mathrm{GHz}$ methanol maser is regularly detected (e.g., [8]), no estimates of the magnetic field strength have been possible due to the unknown Landé g-factors [10]. Very recently, the g-factors for all the methanol maser transitions have been theoretically calculated making possible to estimate a lower limit of the magnetic field strength from the Zeeman-splittings measurements [12].

Here, we present our most recent results on the full polarization observations of $6.7 \mathrm{GHz}$ methanol and $22 \mathrm{GHz}$ water maser emissions made with the European VLBI Network ${ }^{1}$ (EVN). In Sect. 2 we report the updated statistics of the possible alignment between the magnetic field and the bipolar outflows ejected from the massive protostars obtained by analyzing the linearly polarized emission of methanol masers. We will also provide lower limits of the magnetic field strength measured from the Zeeman-splitting of the methanol maser. In Sect. 3 the up-to-date results of the monitoring project of the $22 \mathrm{GHz}$ water masers detected towards the massive star-forming region W75N(B) will be briefly presented.

\section{6.7 GHz methanol masers: The flux-limited sample}

Between 2008 and 2015 we observed with the EVN 30 massive star-forming regions at 6.7

\footnotetext{
${ }^{1}$ The European VLBI Network is a joint facility of European, Chinese, South African and other radio astronomy institutes funded by their national research councils.
} 

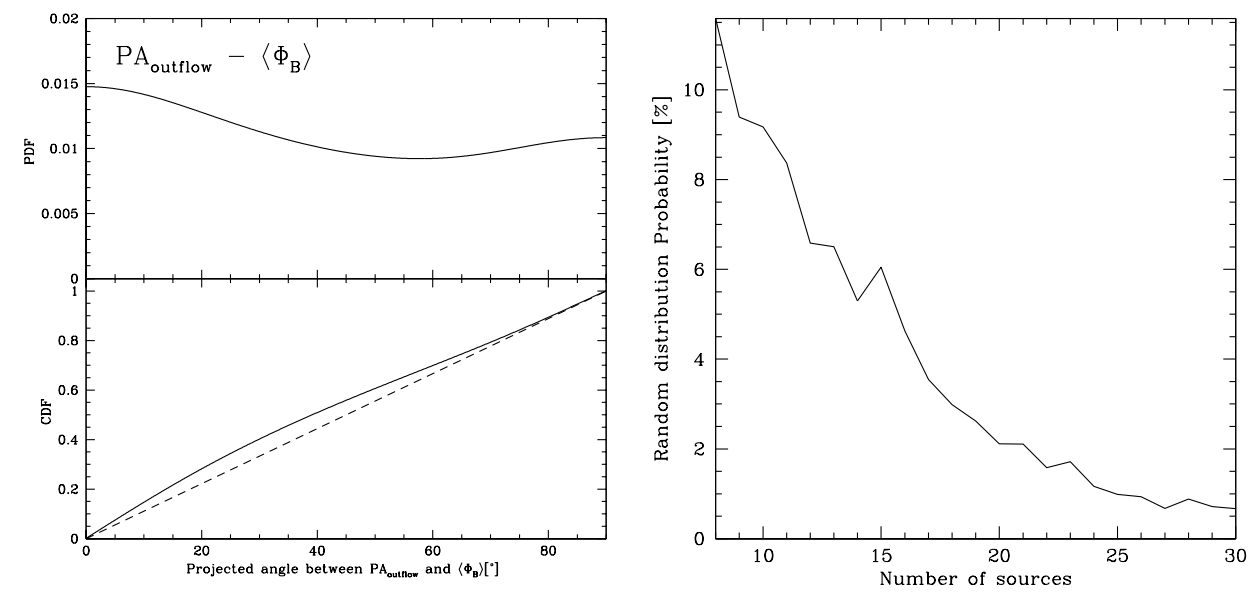

Figure 1: Left. The probability distribution function (PDF, top panel) and the cumulative distribution function (CDF, bottom panel) of the projected angle between the outflow axes and the magnetic field $\left(\left|\mathrm{PA}_{\text {outflow }}-\left\langle\Phi_{\mathrm{B}}\right\rangle\right|\right)$ based on 25 sources as measured by [19]. The dashed line is the $\mathrm{CDF}$ for random orientation of outflows and magnetic fields, i.e. all angular differences are equally likely. Right. The random distribution probability of $\left|\mathrm{PA}_{\text {outflow }}-\left\langle\Phi_{\mathrm{B}}\right\rangle\right|$ determined with a Kolmogorov-Smirnov (K-S) test as function of number of sources [11].

$\mathrm{GHz}$ in full polarization mode to determine both the orientation and the strength of magnetic field at mas resolution by analyzing the polarized emission of methanol masers. The sources were selected from [13] accordingly to their declination $\left(>-9^{\circ}\right)$ and to their methanol maser singledish flux density ( $>50 \mathrm{Jy}$ ). To ensure polarization detection we exclude sources whose single-dish flux density decreased below $20 \mathrm{Jy}[10]$.

We detected a total of $762 \mathrm{CH}_{3} \mathrm{OH}$ maser features towards the 25 sources analyzed so far $[8,14,15,16,17,18,19], 25 \%$ and $4 \%$ of which showed linearly (with linear polarization fraction $\left.P_{1}=0.3 \%-17.0 \%\right)$ and circularly polarized emission $\left(P_{\mathrm{V}}=0.1 \%-0.5 \%\right)$, respectively. Only in one case no polarized emission was measured (G174.20-0.08; [8]). From the analysis of the linearly polarized emission, which was performed by using the adapted Full Radiative Transfer Method (FRTM) code for $6.7 \mathrm{GHz}$ methanol masers [20, 15] modified as described in [19], we measured well ordered magnetic field vectors within the same source. The magnetic field is found to be parallel to the molecular outflow (misalignment $\left.<30^{\circ}\right)$ in 13 massive YSOs $(\sim 50 \%$ of the analyzed sample) and perpendicular $\left(>75^{\circ}\right)$ in seven massive YSOs [19]. Furthermore, where a comparison of the magnetic field at small and large scales was possible (W51 and IRAS 20126+4104), we found a perfect agreement of its morphology from 10 au to 1000 au $[16,18]$.

\subsection{Up-to-date statistics}

To determine if there exists any relation between the morphology of the magnetic field and the ejection direction of molecular outflows from massive YSOs on tens-au scales, we performed a Kolmogorov-Smirnov (K-S) test on the 25 sources for which we were able to measure on the plane of the sky the angle between the magnetic field orientation $\left(\left\langle\Phi_{\mathrm{B}}\right\rangle\right)$ and the outflows axis $\left(\mathrm{PA}_{\text {outflow }}\right)$. Among these 25 sources two were observed in the Southern Hemisphere by [21]. The test shows a probability of $3 \%$ that the distribution of the projected angles $\left|\mathrm{PA}_{\text {outflow }}-\left\langle\Phi_{\mathrm{B}}\right\rangle\right|$ is drawn from a random distribution (see Fig. 1). This finding is consistent with our prediction obtained by simu- 
lating, via Monte-Carlo, a randomly selected sample of vectors in a pair representing $\mathrm{PA}_{\text {outflow }}$ and $\left\langle\Phi_{\mathrm{B}}\right\rangle$, with the only apriori condition that their $3 \mathrm{D}$ misalignment is $\left\langle 30^{\circ}\right.$ (see Fig. 1; [8]). Even if a deeper statistical analysis is necessary, we have a statistical evidence that the magnetic fields around massive YSOs seems to be preferentially oriented along the molecular outflows. Nevertheless, a quite large number of sources $(\sim 30 \%)$ show a projected magnetic field perpendicular to the outflows axis.

\subsection{Magnetic field strength}

Until 2017 the estimation of the magnetic field strength from the Zeeman-splitting measurements was impossible due to the uncertainty of the Landé g-factors for the methanol maser transitions. We already discussed in [11] the difficulties for determining the g-factors from laboratory measurements of the Zeeman-splittings of the complex methanol molecule. However, the g-factors can be estimated by theoretically modeling the entire Zeeman effect of the methanol molecule. This was done by Lankhaar et al. [22, 12] who modeled the hyperfine structure of the torsion-rotation transitions responsible of the maser lines. The model shows that each single methanol maser line is composed of several hyperfine transitions $(>8)$ with frequency separations of the order of few $\mathrm{kHz}$ (Tables I-XIV of [22]). Actually, it is still unknown how much a hyperfine transition contributes to its maser emission, therefore a detailed pumping model is absolutely fundamental to resolve this issue. However, assuming that the hyperfine transition with the largest Einstein coefficient for stimulated emission, i.e. $F=3 \rightarrow 4$, is more favored among the eight hyperfine transitions that might contribute to the maser line, we can provide lower limit of $B_{\|}=\frac{\Delta V_{Z}}{\alpha_{Z}^{3 \rightarrow 4}}$ from our Zeeman-splitting measurements $\left(\Delta V_{Z}\right)$. The Zeeman-splitting coefficient is $\alpha_{Z}^{3 \rightarrow 4}=-0.051 \mathrm{~km} \mathrm{~s}^{-1} \mathrm{G}^{-1}$ [12]. Unfortunately, the $\Delta V_{Z}$ reported in our previous papers ([15, 16, 17, 18, 8]) were overestimated due to some inaccuracies in the FRTM code used at that time. We modelled with the updated and correct FRTM code the circularly polarized emission of the methanol maser features detected so far. We measured lower limits of $\left|B_{\|}\right|>5 \mathrm{mG}$ towards all the sources, with the exception of W3(OH) for which we estimated a lower limit of the order of few hundreads $\mathrm{mG}$. More details are reported in [19].

\section{3. $22 \mathrm{GHz}$ water masers: monitoring project of $\mathrm{W} 75 \mathrm{~N}(\mathrm{~B})$}

From 1999 to 2012 we have monitored at VLBI scale the expansion of a water maser shell around an unresolved continuum source excited by the massive YSO W75N-VLA2 [23, 24, 25, 9]. We found that the water maser shell expands at about 5 mas/yr and evolved from an almost circular wind-driven shell to an elliptical morphology. We interpreted this as a "real time" transition from a non-collimated outflow event into a collimated outflow/jet structure during the early stages of evolution of a massive YSO [9]. From the analysis of the polarized emission of the masers we measured that the magnetic field in 7 years (from 2005 to 2012) changed its orientation following the rotation of the major-axis of the elliptical structure and decreased its strength [24, 9]. These measurements are unique and this behavior of the magnetic field has never been observed towards a massive YSO so far. In 2014 we started an EVN monitoring project (four epochs separated by two years from one another) with the aim to follow both the expansion of the outflow/jet structure around VLA 2 and, more importantly, the variation of the magnetic field in the region. The first 

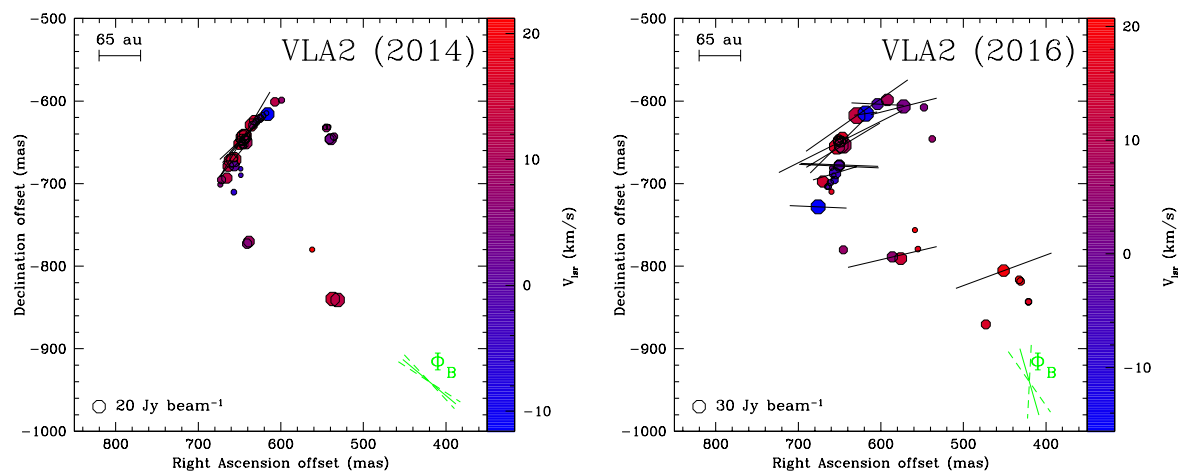

Figure 2: A close-up view of the $22 \mathrm{GHz} \mathrm{H}_{2} \mathrm{O}$ maser features detected with the EVN around the radio sources VLA 2 in epochs 2014.46 (left panels) and 2016.45 (right panels). The reference position in epoch 2014.46 is $\alpha_{2000}=20^{\mathrm{h}} 38^{\mathrm{m}} 36^{\mathrm{s}} .43399$ and $\delta_{2000}=42^{\circ} 37^{\prime} 34^{\prime \prime} .8710$; while in epoch 2016.45 is $\alpha_{2000}=20^{\mathrm{h}} 38^{\mathrm{m}} 36^{\mathrm{s}} .43403$ and $\delta_{2000}=42^{\circ} 37^{\prime} 34^{\prime \prime} .8667$. The octagonal symbols are the identified maser features. The linear polarization vectors, scaled logarithmically according to polarization fraction $P_{1}$, are overplotted. In the bottom-right corner the error-weighted orientation of the magnetic field $\left(\Phi_{\mathrm{B}}\right)$ is also reported; the two dashed segments indicate the uncertainties.

three epochs were observed in 2014, 2016, and 2018. The results of the first two epochs confirm the immutable maser distribution and magnetic field orientation around VLA 1 . While the maser distribution around VLA 2 did not change, the magnetic field changed again (see Fig. 2). In 2014 it was perfectly in agreement with the orientation measured in 2012, while in 2016 the magnetic field rotated back to its 2005 orientation (see Fig. 2). This behaviour of the magnetic field is unexpected. The next two EVN epochs (2018 and 2020) will hopefully help to clarify the phenomenon. Also the magnetic field strength around VLA 2 increased by a factor of 15 since 2012 [9]. This could be due to a further compression of the gas at the shock front caused by the encounter with a much denser medium than in the past.

\section{References}

[1] J.C. Tan, M.T. Beltrán, P. Caselli et al., Massive Star Formation, Protostars and Planets VI, Henrik Beuther, Ralf S. Klessen, Cornelis P. Dullemond, and Thomas Henning (eds.), University of Arizona Press, Tucson (2014) 149 []arXiv:1402.0919]

[2] Y. Matsushita, Y. Sakurai, T. Hosokawa et al., Massive outflows driven by magnetic effects - II. Comparison with observations, MNRAS, 475 (2018) 391 [arXiv:1711.05910]

[3] R. Banerjee, \& R.E. Pudritz Massive Star Formation via High Accretion Rates and Early Disk-driven Outflows, ApJ 660 (2007) 479 [arXiv:astro-ph/0612674]

[4] D. Seifried, R.E. Pudritz, R. Banerjee et al., Magnetic fields during the early stages of massive star formation - II. A generalized outflow criterion, MNRAS, 422 (2012) 347 [arXiv:1109.4379].

[5] Y. Matsushita, M.N. Machida, Y. Sakurai et al., Massive outflows driven by magnetic effects in star-forming clouds with high mass accretion rates, MNRAS 470 (2017) 1026 [arXiv:1704.03185] 
[6] P. Hennebelle, B. Commerçon, M. Joos et al., Collapse, outflows and fragmentation of massive, turbulent and magnetized prestellar barotropic cores, A\&A 528 (2011) A72 [arXiv:1101.1574]

[7] Q. Zhang, K. Qiu, J.M. Girart et al., Magnetic Fields and Massive Star Formation, ApJ, 792 (2014) 116 [arXiv:1407.3984]

[8] G. Surcis, W.H.T. Vlemmings, H.J. van Langevelde et al., EVN observations of 6.7 GHz methanol maser polarization in massive star-forming regions III. The flux-limited sample., A\&A,578 (2015) A102. [arXiv:1504.06325]

[9] G. Surcis, W.H.T. Vlemmings, H.J. van Langevelde et al., Rapidly increasing collimation and magnetic field changes of a protostellar H2O maser outflow, A\&A 565L(2014) 8S [arXiv:1405.1422]

[10] W.H.T. Vlemmings, R.M. Torres \& R. Dodson, Zeeman splitting of 6.7 GHz methanol masers. On the uncertainty of magnetic field strength determinations, A\&A, 529 (2011) A95.

[11] G. Surcis, W.H.T. Vlemmings, H.J. van Langevelde et al., Magnetic field measurements at milliarcsecond resolution around massive young stellar objects, in proceeding of 12th European VLBI Network Symposium and Users Meeting POS (EVN 2014) 041 (2014)

[12] B. Lankhaar, W.H.T. Vlemmings, G. Surcis et al., Characterization of methanol as a magnetic field tracer in star-forming regions, NatAs 2 (2018) 145 [arXiv:1802.05764]

[13] M.R. Pestalozzi, V. Minier \& R.S. Booth, A general catalogue of 6.7-GHz methanol masers. I. Data, $A \& A, 432$ (2005) 737 [arXiv:astro-ph/0411564].

[14] G. Surcis, W.H.T. Vlemmings, R. Dodson et al., Methanol masers probing the ordered magnetic field of W75N, A\&A, $\mathbf{5 0 6}$ (2009) 757 [arXiv:0908.3585].

[15] G. Surcis, W.H.T. Vlemmings, R.M. Torres et al., The properties and polarization of the H2O and CH3OH maser environment of NGC 7538-IRS 1 A\&A, 533A (2011) 47 [arXiv:1107.5313].

[16] G. Surcis, W.H.T. Vlemmings, H.J. van Langevelde et al., EVN observations of 6.7 GHz methanol maser polarization in massive star-forming regions, $A \& A$, 541A (2012) 47 [arXiv:1203.4566].

[17] G. Surcis, W.H.T. Vlemmings, H.J. van Langevelde et al., EVN observations of 6.7 GHz methanol maser polarization in massive star-forming regions II. First statistical results. A\&A, 556 (2013) 73 [arXiv:1306.6335].

[18] G. Surcis, W.H.T. Vlemmings, H.J. van Langevelde et al., The magnetic field at milliarcsecond resolution around IRAS 20126+4104 A\&A, 563 (2014) A30 [arXiv:1401.7987].

[19] G. Surcis, W.H.T. Vlemmings, H.J. van Langevelde et al., 2019, A\&A, accepted

[20] W.H.T. Vlemmings, G. Surcis, K.J.E. Torstensson et al., Magnetic field regulated infall on the disc around the massive protostar CepheusAHW2, MNRAS, 404 (2010) 134 [arXiv:1002.2214].

[21] R. Dodson \& C.D. Moriarty, Probing the magnetic fields of massive star-forming regions with methanol maser polarization, MNRAS, 421 (2012) 2395 [arXiv:1201.1687]

[22] B. Lankhaar, G.C. Groenenboom, \& A. van der Avoird Hyperfine interactions and internal rotation in methanol, J Chem Phys 145 (2016) 24

[23] J.M. Torrelles, N.A. Patel, G. Anglada et al., Evidence for Evolution of the Outflow Collimation in Very Young Stellar Objects ApJ, 598 (2003) 115

[24] G. Surcis, W.H.T. Vlemmings, S. Curiel et al., The structure of the magnetic field in the massive star-forming region W75N A\&A, 527 (2011) 48 [arXiv:1101.1956].

[25] J.-S. Kim, S.-W. Kim; T. Kurayama et al., Evolution of the Water Maser Expanding Shell in W75N VLA 2, ApJ 767 (2013) 86 [arXiv:1303.1686] 